\title{
Stability and Boundedness of Solutions of Certain Non-Autonomous Third Order Nonlinear Differential Equations
}

\author{
Akinwale L. Olutimo, Folashade 0. Akinwole \\ Department of Mathematics, Lagos State University, Ojo, Nigeria \\ Email: aolutimo@yahoo.com, gbolasade005@yahoo.com \\ Received 6 December 2015; accepted 23 January 2016; published 27 January 2016 \\ Copyright (C) 2016 by authors and Scientific Research Publishing Inc. \\ This work is licensed under the Creative Commons Attribution International License (CC BY). \\ http://creativecommons.org/licenses/by/4.0/ \\ cc) (i) \\ Open Access
}

\begin{abstract}
In this paper, by defining an appropriate Lyapunov functional, we obtain sufficient conditions for which all solutions of certain real non-autonomous third order nonlinear differential equations are asymptotically stable and bounded. The results obtained improve and extend some known results in the literature.
\end{abstract}

\section{Keywords}

Nonlinear Differential Equations, Third Order, Asymptotic Stability, Boundedness, Lyapunov Method

\section{Introduction}

We shall be concerned here, with stability and boundedness of solutions of the third order, non-linear, nonautonomous differential equation of the form:

$$
\dddot{x}+a(t) \phi(x, \dot{x}) \ddot{x}+b(t) f(x, \dot{x})=p(t)
$$

where $a(t), b(t)$ are positive continuously differentiable functions and $\phi, f$ and $p$ are continuous real-valued functions depending only on the arguments shown, and the dots indicate the differentiation with respect to $t$. Moreover, the existence and uniqueness of solutions of (1.1) will be assumed.

The Lyapunov function or functional approach has been a powerful tool to ascertain the stability and boundedness of solutions of certain differential equations. Up to now, perhaps, the most effective method to determine the stability and boundedness of solutions of non-linear differential equations is still the Lyapunov's direct (or second) method. The major advantage of this method is that stability in the large and boundedness of 
solutions can be obtained without any prior knowledge of solutions. Today, this method is widely recognized as an excellent tool not only in the study of differential equations but also in the theory of control systems, dynamical systems, systems with time lag, power system analysis, time varying non-linear feedback systems, and so on. Its chief characteristic is the construction of a scalar function or functional, namely, the Lyapunov function or functional. This function or functional and its time derivative along the system under consideration must satisfy some fundamental inequalities. But, finding an appropriate Lyapunov function or functional is in general a difficult task. See [1].

Stability analysis and boundedness of solutions of nonlinear systems are important area of current research and many concept of stability and boundedness of solutions have in the past been studied by several authors. See for instance, a survey book, Ressig et al. [2] and in a sequence of results by [3]-[8]. With respect to our observation in the relevant literature, these authors consider stability, asymptotic behavior and boundedness of solutions of Equation (1.1) for which $\phi(x, \dot{x})$ equals any of $f(x, \dot{x}, \ddot{x}), f(x, \dot{x}), f(x)$ and $f(x, \dot{x})$ equals $g(\dot{x})+h(x)$, with $a(t)=b(t)=1$. The special case for which $\phi(x, \dot{x})=f(x, \dot{x}, \ddot{x}), f(x, \dot{x}), f(x)$ and $f(x, \dot{x})=g(\dot{x})+h(x)$ with $a(t) \neq 1, b(t) \neq 1$ have received little attention due to the difficulty in constructing suitable scalar function. For example, see [9]-[12]. However, no work based on (1.1) was found. The result here will be different from those mentioned.

The motivation for the present work is derived from the papers of the authors mentioned above. Our aim is to extend their results to the very special case in Equation (1.1) for the boundedness and asymptotic behavior of solutions.

\section{Statement of Results}

Our main results are the following theorems.

Theorem 1 Suppose $a(t), b(t)$ are continuously differentiable on $[0, \infty)$ and $f(0,0)=0$ and the following conditions are satisfied;

(i) $a(t) \geq a_{o}>0, \quad b(t) \geq b_{o}>0$ for $t \in I=[0, \infty)$;

(ii) $\phi(x, y) \geq a, f_{y}(x, y) \geq b, f_{x}(x, 0) \geq c, a b-c>0$ for all $x, y$;

(iii) $f_{z}(x, 0) \geq 0, y \int_{0}^{y} f_{x}(x, y) \mathrm{d} y \leq 0, \quad y \int_{0}^{y} \phi_{x}(x, y) \mathrm{d} y \leq 0$, for all $x, y$ and $\frac{f(x, 0)}{y} \geq \gamma, y \neq 0 \quad$;

(iv) $\limsup _{(t, v) \rightarrow(\infty, \infty)} \frac{1}{V} \int_{t}^{t+v}\left\{\left|a^{\prime}(s)\right|+\left|b^{\prime}(s)\right|\right\} \mathrm{d} s<\epsilon$, where $\epsilon$ is a small positive constant whose magnitude depends only on the constants appeared in (i)-(iii).

Then, every solution $x \equiv x(t)$ of (1.1) is asymptotically stable and satisfies

$$
x(t) \rightarrow 0, \quad \dot{x}(t) \rightarrow 0, \quad \ddot{x}(t) \rightarrow 0 \text { as } t \rightarrow \infty .
$$

Theorem 2 Let all the conditions of Theorem 1 be satisfied, and in addition we assume that there exist a finite constant $\delta_{o}>0$ and a non-negative and continuous function $q(t)$ such that $p$ satisfies

(i) $|p(t)| \leq q(t)$,

where $\int_{0}^{t} q(s) \mathrm{d} s=\delta_{o}<\infty$ for all $t>0$.

Then every solution $x(t)$, of (1.1) satisfies

$$
|x(t)| \leq D, \quad|\dot{x}(t)| \leq D, \quad|\ddot{x}(t)| \leq D
$$

for all sufficiently large $t$, while $D$ is a finite constant.

Remark 2.1 Our results develop Qian [13], Omeike [14] and Tunc's [15] results to the non-autonomous of the form (1.1).

It is convenient here to consider, the equivalent system of (1.1);

$$
\begin{aligned}
& \dot{x}=y, \\
& \dot{y}=z,
\end{aligned}
$$




$$
\dot{z}=-a(t) \phi(x, y) z-b(t) f(x, y)+p(t)
$$

and show that under the conditions stated in the theorem, every solution $(x(t), y(t), z(t))$ of (2.2) satisfies

$$
|x(t)| \leq D, \quad|y(t)| \leq D, \quad|z(t)| \leq D
$$

for all sufficiently large $t$, where $D$ is the constant in (2.1).

Our proof of (2.3) rests entirely on the lemma stated below and the scalar function $V=V(t, x, y, z)$ defined by

$$
\begin{aligned}
2 V= & 2 \alpha b(t) \int_{0}^{x} f(\xi, 0) \mathrm{d} \xi+2 b(t) f(x, 0) y+2 \alpha a(t) \int_{0}^{y} \phi(x, \eta) \eta \mathrm{d} \eta \\
& +2 b(t) \int_{0}^{y} f(x, \eta) \mathrm{d} \eta+2 \alpha y z+z^{2},
\end{aligned}
$$

and $\alpha>0$, an arbitrary fixed constant such that

$$
\frac{c}{b}<a<\alpha<a_{o} a .
$$

Lemma 1 Subject to the conditions of Theorem 1 there are positive constants $D_{1}, D_{2}, D_{3}, D_{4}$, and $D_{5}$ depending only on $a, b, c, a_{o}, b_{o}$, and $\alpha$ such that

$$
V(t, x, y, z) \geq D_{1}\left(x^{2}+y^{2}+z^{2}\right)
$$

Furthermore, there are finite constants $D_{2}>0, D_{3}>0$ dependent only $a, b, c, a_{o}, b_{o}, \delta_{o}$ and $\alpha$ such that any solution $(x(t), y(t), z(t))$ of (2.2),

$$
\dot{V} \equiv \frac{\mathrm{d}}{\mathrm{d} t} V(t, x(t), y(t), z(t)) \leq-D_{2}
$$

provided that $y^{2}+z^{2} \geq D_{3}$.

Proof: To verify (2.6) observe first that the expressions $2 V$ in (2.4) may be re-arranged in the form,

$$
\begin{aligned}
2 V= & (\alpha y+z)^{2}+\alpha \frac{b(t)}{a b}\left\{f(x, 0)+a \alpha^{-1} b y\right\}^{2}+2 \alpha \int_{o}^{y}\{a(t) \phi(x, y)-\alpha\} \eta \mathrm{d} \eta \\
& +\frac{2}{\alpha} b(t) \int_{o}^{y}\{\alpha f(x, y)-a b\} \eta \mathrm{d} \eta+2 \alpha \frac{b(t)}{a b} \int_{o}^{x}\left[a b-f_{\xi}(\xi, 0)\right] f(\xi, 0) \mathrm{d} \xi-f^{2}(0,0) .
\end{aligned}
$$

By conditions (ii) of Theorem 1 and $f(0,0)=0$, we have that the term

$$
2 \alpha \frac{b(t)}{a b} \int_{o}^{x}\left[a b-f_{\xi}(\xi, 0)\right] f(\xi, 0) \mathrm{d} \xi-f^{2}(0,0)
$$

in the re-arrangement of $2 \mathrm{~V}$ becomes

$$
2 \alpha \frac{b(t)}{a b} \int_{o}^{x}\left[a b-f_{\xi}(\xi, 0)\right] f(\xi, 0) \mathrm{d} \xi-f^{2}(0,0) \geq \alpha \frac{b_{o}}{a b}(a b-c) c x^{2} .
$$

Since $f_{x}(x, 0) \geq c, f_{y}(x, y) \geq b$ and $\phi(x, y) \geq a$, (i) of Theorem 1 and combining all these with (2.8), we have

$$
\begin{aligned}
2 V \geq & \alpha \frac{b_{o}}{a b}(a b-c) c x^{2}+\left\{\alpha\left(a_{o} a-\alpha\right)+\frac{b_{o}}{\alpha}(\alpha b-a b)\right\} y^{2} \\
& +(\alpha y+z)^{2}+\alpha \frac{b_{o}}{a b}\left\{f(x, 0)+a \alpha^{-1} b y\right\}^{2},
\end{aligned}
$$

for all $x, y$ and $z$. Since $\alpha$ satisfy (2.5) and $a b-c>0$, the constants $a_{o} a-\alpha$ and $\alpha b-a b$ are positive. This implies that there exists a constant small enough such that

$$
D_{4}=\min \left\{\alpha \frac{b_{o}}{a b}(a b-c) c ; \alpha\left(a_{o} a-\alpha\right)+\frac{b_{o}}{\alpha}(\alpha b-a b), 1\right\}
$$


we have

$$
V(t, x, y, z) \geq D_{4}\left(x^{2}+y^{2}+z^{2}\right) .
$$

Next, we prove the inequality (2.7). Along any solution $(x(t), y(t), z(t))$ of (2.2), we have

$$
\begin{aligned}
\dot{V}= & b(t) y^{2} f_{x}(x, 0)+b(t) y \int_{o}^{y} f_{x}(x, \eta) d \eta+b(t) f(x, 0) z \\
& -\alpha b(t)\{f(x, y)-f(x, 0)\} y-\{a(t) \phi(x, y)-\alpha\} z^{2} \\
& +\alpha b^{\prime}(t) \int_{o}^{x} f(\xi, 0) \mathrm{d} \xi+b^{\prime}(t) f(x, 0) y+\alpha a^{\prime}(t) \int_{o}^{y} \phi(x, \eta) \eta \mathrm{d} \eta \\
& +b^{\prime}(t) \int_{o}^{y} f(x, \eta) \mathrm{d} \eta .
\end{aligned}
$$

We easily see that by hypothesis (ii) of Theorem 1 ,

$$
\begin{gathered}
\alpha b(t)\{f(x, y)-f(x, 0)\} y=\alpha b(t)\left\{f_{y}(x, \theta y)\right\} y^{2}, \quad 0 \leq \theta \leq 1 \\
\geq \alpha b_{o} b y^{2} \\
\{a(t) \phi(x, y)-\alpha\} z^{2} \geq\left(a_{o} a-\alpha\right) z^{2}
\end{gathered}
$$

and

$$
b(t) y^{2} f_{x}(x, 0) \geq b_{o} c y^{2} .
$$

By hypothesis (iii)

$$
\begin{gathered}
\alpha a(t) y \int_{o}^{y} \phi_{x}(x, \eta) \eta \mathrm{d} \eta \leq \alpha a_{o} y \int_{o}^{y} \phi_{x}(x, \eta) \eta \mathrm{d} \eta \leq 0, \\
b(t) y \int_{o}^{y} f_{x}(x, \eta) \mathrm{d} \eta \leq b_{o} y \int_{o}^{y} f_{x}(x, \eta) \mathrm{d} \eta \leq 0 .
\end{gathered}
$$

Also,

$$
b(t) f(x, 0) z=b(t)\left\{f_{z}(x, 0)\right\} z^{2} \geq 0
$$

Thus,

$$
\dot{V} \leq-b_{o}(\alpha b-c) y^{2}-\left(a_{o} a-\alpha\right) z^{2}+k\left[\left|a^{\prime}(t)+b^{\prime}(t)\right|\right]\left(y^{2}+z^{2}\right)
$$

that is,

$$
\dot{V} \leq-\delta_{1}\left(y^{2}+z^{2}\right)+k\left[\left|a^{\prime}(t)+b^{\prime}(t)\right|\right]\left(y^{2}+z^{2}\right)
$$

where $\delta_{1}=\min \left\{b_{o}(\alpha b-c),\left(a_{o} a-\alpha\right)\right\}$ and $k=\max \{\alpha a,(\gamma+\alpha c)\}$ are constants.

Using the inequality (2.6) for all $(x, y, z) \in \mathbb{R}^{3}$ and $0 \leq t \in \mathbb{R}^{+}$, we have that

$$
\dot{V} \leq-\left\{\delta_{1}-k\left[\left|a^{\prime}(t)+b^{\prime}(t)\right|\right]\right\} V(t, x, y, z)
$$

Thus,

$$
\dot{V} \leq-\left\{\delta_{1}-k \phi(t)\right\} V(t, x, y, z)
$$

let

$$
\phi(t)=\lim \sup _{(t, v) \rightarrow(\infty, \infty)} \frac{1}{V} \int_{t}^{t+v}\left\{\left|a^{\prime}(s)\right|+\left|b^{\prime}(s)\right|\right\} \mathrm{d} s<\delta_{1} k^{-1} .
$$

Just as in (2.7), we obtain

$$
\dot{V} \leq-D_{5} .
$$

Proof of Theorem 1: It follows that $\frac{\mathrm{d}}{\mathrm{d} t} V\left(x_{t}, y_{t}, z_{t}\right)=0$ if and only if $x_{t}=y_{t}=z_{t}=0$. 
Thus, in view of (2.9) and (2.10) and the last discussion, it shows that the trivial solution of (1.1) is asymptotically stable.

Hence, the proof of Theorem 1 is complete.

Proof of Theorem 2: The proof of Theorem 2 depends on the scalar differentiable Lyapunov function $V(x, y, z)$ defined in (2.4).

For $p(t) \neq 0$,

$$
\dot{V}(x, y, z)=\dot{V}_{(2.2)}(x, y, z)+(\alpha y+z)|p(t)|
$$

Since $\dot{V}_{(2.2)} \leq 0$ in (2.11) for all $t, x, y, z$ thus

$$
\dot{V}(x, y, z) \leq-\delta_{2}\left(y^{2}+z^{2}\right)+(\alpha|y|+|z|) q(t)
$$

Hence, it follows that

$$
\dot{V}(x, y, z) \leq-\delta_{2}\left(y^{2}+z^{2}\right)+\delta_{3}(|y|+|z|) q(t) \leq \delta_{3}(|y|+|z|) q(t)
$$

for a constant $\delta_{3}>0$, where $\delta_{3}=\max \{\alpha, 1\}$.

Making use of the inequalities $|y|<1+y^{2}$ and $|z|<1+z^{2}$. It is clear that

$$
\dot{V}(x, y, z) \leq \delta_{3}\left(2+y^{2}+z^{2}\right) q(t)
$$

by (2.6), we have $y^{2}+z^{2} \leq\left(x^{2}+y^{2}+z^{2}\right) \leq D_{1}^{-1} V(x, y, z)$.

Hence,

$$
\dot{V}(x, y, z) \leq \delta_{3}\left(2+D_{1}^{-1} V(x, y, z)\right) q(t)
$$

or

$$
\dot{V}(x, y, z) \leq \delta_{3} q(t) D_{1}^{-1} V(x, y, z)+2 \delta_{3} q(t) .
$$

We integrate both sides of this inequality from 0 to $t$ and using Gronwall-Bellman inequality, we obtain

$$
\begin{aligned}
V & \leq V(x(0), y(0), z(0))+2 \delta_{3} \delta_{o}+\delta_{3} D_{1}^{-1} \int_{0}^{t} V(x(s), y(s), z(s)) q(s) \mathrm{d} s, \\
& \leq\left(V(0,0,0)+2 \delta_{3} \delta_{o}\right) \exp \left(\delta_{3} D_{1}^{-1} \int_{0}^{t} q(s) \mathrm{d} s\right) \\
& =\left(V(0,0,0)+2 \delta_{3} \delta_{o}\right) \exp \left(\delta_{3} D_{1}^{-1} \delta_{o}\right)=D<\infty
\end{aligned}
$$

where $D>0$ is a constant and $\delta_{o}=\int_{0}^{t} q(s) \mathrm{d} s$.

Now, since the right-hand side is a constant and since $V(x, y, z) \rightarrow \infty$ as $x^{2}+y^{2}+z^{2} \rightarrow \infty$, it follows that there exist a constant $D>0$ such that

$$
|x(t)| \leq D, \quad|y(t)| \leq D, \quad|z(t)| \leq D \quad \text { for } t \geq 0 .
$$

From the system (1.1), this implies that

$$
|x(t)| \leq D, \quad|\dot{x}(t)| \leq D, \quad|\ddot{x}(t)| \leq D \quad \text { for } t \geq 0 .
$$

The proof of Theorem 2 is now complete.

\section{Conclusions}

The solutions of the third-order non-autonomous nonlinear system are bounded and asymptotically stable according to the Lyapunov's theory if the inequality (2.5) is satisfied.

Example 2.1 We consider a certain third order non-autonomous scalar differential equation of the form

$$
\dddot{x}+2 \tan t\left[\cos (x) \dot{x}+(\dot{x})^{2}+3\right] \ddot{x}+3 \sin \left(2 t^{2}\right)\left[(\dot{x})^{3}+\dot{x}+x+\frac{x}{1+x^{2}}\right]=\frac{1}{2+2 t^{2}}, \quad t \in I=[0, \infty) .
$$




$$
\begin{aligned}
& \dot{x}=y, \\
& \dot{y}=z, \\
& \dot{z}=-2 \tan t\left[\cos (x) y+y^{2}+3\right] z-3 \sin \left(2 t^{2}\right)\left[y^{3}+y+x+\frac{x}{1+x^{2}}\right]+\frac{1}{2+2 t^{2}} . \\
& a(t)=2 \tan t \\
& b(t)=3 \sin \left(2 t^{2}\right) \\
& \psi(x, y)=\cos (x) y+y^{2}+3 \\
& f(x, y)=y^{3}+y+x+\frac{x}{1+x^{2}} \\
& p(t)=\frac{1}{2+2 t^{2}} \\
& |a(t)|=|2 \tan t| \leq 2=a_{o} \\
& |b(t)|=\left|3 \sin \left(2 t^{2}\right)\right| \leq 3=b_{o} \\
& \psi(x, y)=\cos (x) y+y^{2}+3 \geq-|\cos x||y|+y^{2}+3 \\
& \geq|y|+y^{2}+3=\left(|y|+\frac{1}{2}\right)^{2}+\frac{11}{4}>2=a \\
& f_{y}(x, y)=3 y^{3}+1 \geq 1=b \\
& f_{x}(x, 0)=1+\frac{1-x^{2}}{\left(1+x^{2}\right)^{2}} \geq 1=c \\
& y \int_{0}^{y} f_{x}(x, v) \mathrm{d} v=y^{2}+\frac{y^{2}}{\left(1+x^{2}\right)^{2}}-\frac{x^{2} y^{2}}{\left(1+x^{2}\right)^{2}} \leq 0 \\
& y \int_{0}^{y} \psi_{x}(x, v) \mathrm{d} v=-(\sin x) \frac{y^{4}}{4} \leq 0 . \\
& \frac{f(x, 0)}{y}=\frac{x\left(1+\frac{1}{1+x^{2}}\right)}{y} \geq \frac{3}{2}=\gamma \text {. }
\end{aligned}
$$

Choosing $\alpha=3$, then, $\delta_{1}=\min \{1,6\}=1$ and $k=\max \left\{\frac{9}{2}, 6\right\}=6$.

Thus,

$$
\limsup _{(t, v) \rightarrow(\infty, \infty)} \frac{1}{V} \int_{t}^{t+v}\left\{\left|2 \sec ^{2}(s)\right|+\left|12 s \cos \left(2 s^{2}\right)\right|\right\} \mathrm{d} s<\frac{1}{6}
$$

and finally,

$$
p(t)=\frac{1}{2+2 t^{2}}
$$

and

$$
\int_{0}^{\infty} q(s) \mathrm{d} s=\frac{1}{2} \int_{0}^{\infty} \frac{1}{1+s^{2}} \mathrm{~d} s=\frac{\Pi}{4}<\infty .
$$


Thus, all conditions of the Theorems are satisfied. Therefore, all solutions of (3.1) are asymptotically stable and bounded.

\section{References}

[1] Chin, P.S.M. (1989) Stability Results for the Solution of Certain Fourth Order Autonomous Differential Equations. International Journal of Control, 49, 1163-1173. http://dx.doi.org/10.1080/00207178908559698

[2] Reissig, R., Sansone, G. and Conti, R. (1974) Nonlinear Differntial Equations of Higher Order. Noordhoff International Publication, Leyden.

[3] Chukwu, E.N. (1975) On the Boundedness of Solutions of Third Order Differential Equations. Annali di Matematica Pura ed Applicata, 104, 123-149. http://dx.doi.org/10.1007/BF02417013

[4] Ezeilo, J.O.C. (1967) A Generalization of a Boundedness Theorem for a Certain Third-Order Differential Equation. Mathematical Proceedings of the Cambridge Philosophical Society, 63, 735-742. http://dx.doi.org/10.1017/S0305004100041736

[5] Harrow, M. (1968) Further Results for the Solutions of Certain Third-Order Differential Equations. Journal London Mathematical Society, 43, 587-592. http://dx.doi.org/10.1112/jlms/s1-43.1.587

[6] Omeike, M.O. (2007) Further Results on Global Stability of Third Order Nonlinear Differential Equations. Nonlinear Analysis, 67, 3394-3400. http://dx.doi.org/10.1016/j.na.2006.10.021

[7] Omeike, M.O. (2008) New Result in the Ultimate Boundedness of Solutions of a Third Order Non-Linear Ordinary Differential Equation. Journal of Inequalities in Pure \& Applied Mathematics, 9, Article 15.8.

[8] Tunc, C. (2010) The Boundedness of Solutions to Nonlinear Third Order Differential Equations. Nonlinear Dynamics and Systems Theory, 10, 97-102.

[9] Hara, T. (1971) On the Stability of Soltutions of Certain Third Ordinary Differential Equations. Proceedings of the Japan Academy, 47, 903-908. http://dx.doi.org/10.3792/pja/1195526293

[10] Hara, T. (1974) On the Asymptotic Behavior of the Solutions of Some Third and Fourth Order Non-Autonomous Differential Equations. Publications of the Research Institute for Mathematical Sciences, 9, 649-673. http://dx.doi.org/10.2977/prims/1195192447

[11] Hara, T. (1975) On the Asymptotic Behavior of Solutions of Certain Non-Autonomous Differential Equations. Osaka Journal of Mathematics, 12, 267-282.

[12] Mehri, B. and Shadman, D. (1999) Boundedness of Solutions of Certain Third Order Differential Equation. Mathematical Inequalities \& Applications, 2, 545-549. http://dx.doi.org/10.7153/mia-02-45

[13] Qian, C. (2000) On Global Stability of Third Order Nonlinear Differential Equations. On Global Stability of Third Order Nonlinear Differential Equations, 42, 651-661.

[14] Omeike, M.O. (2010) New Results on the Asymptotic Behavior of a Third Order Nonlinear Differential Equation. Differential Equations and Applications, 2, 39-51.

[15] Tunc, C. (2005) Boundedness of Solutions of a Third-Order Nonlinear Differential Equations. Journal of Inequalities in Pure \& Applied Mathematics, 6, Article 3, 1-6. 